\title{
RESTORATION OF ANTERIOR TEETH USING AN INDIRECT COMPOSITE TECHNIQUE. CASE REPORT
}

\author{
M. GARGARI ${ }^{1,2}$, F.M. CERUSO ${ }^{2}$, A. PUJIA ${ }^{1,2}$, V. PRETE $^{2}$ \\ ${ }^{1}$ Department of Clinical Science and Translational Medicine, University of Rome "Tor Vergata", Rome, Italy \\ ${ }^{2}$ Department of Dentistry "Fra G.B. Orsenigo - Ospedale San Pietro F.B.F.", Rome, Italy
}

SUMMARY

Objective. This article presents a case report of restoration of anterior teeth using an indirect technique with composite veneers in order to restore the dental anatomy and to provide aesthetic and function of anterior teeth.

Materials and methods. A treatment of upper anterior teeth with indirect micro-hybrid composite restoration was proposed to a 40-year-old woman with old discolored and fractured ceramic veneers. Upper six anterior teeth were prepared and, after impressions with VPS of maxillary arche, the composite veneers were placed.

Discussion and results. There are several advantages offered by indirect techniques compared to direct techniques. The increased practice of veneering technique with indirect composites is due to improvement in the properties of composite materials in the last years. The results are very favourable and the patient is satisfied.

Conclusions. The restorations with composite veneers have proved durable and aesthetic, protect tooth structure and aesthetic and function is predictably re-established.

Key words: veneers, composite, restoration, indirect technique, aesthetic.

\section{Introduction}

Re-establishing a patient's lost natural dental esthetics is among the important topics of today's dentistry, in addition to function and fonation. Color, shape, and structural and position abnormalities of anterior teeth might lead to important aesthetic problems for patients. In order to solve such problems, the technique preferred frequently is to cover the teeth with dental crowns. However, excessive preparations of teeth and damages to surrounding tissues, such as gingiva, are some disadvantages of crowns (1). Therefore, in recent years, laminate veneer restorations, as a more aesthetic and more conservative treatment option, have been used in dentistry (1).

Currently, two types of veneering of alloy frameworks are in use, either veneering with ceramic or with composite. Veneering with ceram- ic is an established technology and has been used for more than 50 years in fixed dental prosthesis for the anterior and posterior regions of the mouth with very good success rate. Veneering ceramics stand out with high biocompatibility, high color stability and high abrasion stability. However, veneering ceramic restorations have numerous undesirable characteristics, such as that the fabrication, is time consuming and technically demanding, and is abrasive for opposing natural tooth structure (2).

The increased practice of veneering technique with indirect composites is due to improvement in the properties of composite materials in the last years. These new composites have a volume percentage of inorganic ceramic fillers of approximately $66 \%$ which result into improved mechanical properties with a flexural strength between 120 and $160 \mathrm{MPa}$ and an elastic modulus of 8.5 -12 GPa. Most veneering composites are 
applied with a post-curing process that result in superior flexural strength compared to veneering ceramic, minimal polymerization shrinkage, and wear rate comparable to tooth enamel (2).

The aim of this study is to present the indirect restorative technique with composite veneers providing esthetic in order to restore the dental anatomy of anterior teeth.

\section{Materials and methods}

A 40-year-old woman with old discolored and fractured veneers come to our observation (Fig. 1). She was in good general health, she doesn't have allergies to medications, she doesn't smoke. No temporomandibular joint pain was referred. Dental hygiene and periodontal conditions were not optimal, so the patient was referred to the periodontist for a hygienic phase before restorative treatment and was instructed to maintain her oral hygiene after the treatment. Following oral hygiene measure, it was decided to restore upper anterior teeth with indirect micro-hybrid composite restoration.

After clinical exam, impressions of maxillary and mandible arches were taken with alginate to obtain preliminary casts for fabrication of 6 provisional veneers in acrylic resin. Then, upper six anterior teeth were prepared by the removal of $0.5-0.75 \mathrm{~mm}$ enamel with a medium grit chamfer bur with proximoincisal shoulder preparation. Further, arch impression of the prepared teeth was made (Fig. 2) using a polyvinylsiloxane impression material (Aquasil Easy Mix Putty, Aquasil Ultra XLV, DENTSPLY Caulk, Milford, USA). For the recording of the beyond preparation have been used 2 different sizes of wires retraction: size " 00 " more deeply in the gingival sulcus and size "1" more superficial. The first wire was moistened with ferric sulfate at $25 \%$ of concentration and the second with aluminum chloride at $20 \%$ of concentration.

The cast is prepared using die-stone. A microhybrid composite (Estelite Sigma Quick, Tokuyama Dental Corporation, 38-9, Taitou 1-chome, Taitou-kuu, Tokyo, Japan) shade A2 was applied and A3 for cervical area and enamel WE. Finishing and polishing of veneers were carried out with disc and cup shaped $3 \mathrm{M}$. Although a significant advantage exists over direct composite veneers, indirect veneers made of processed composite possess limited bond strength because of the reduced potential to form chemical bond with the bonding medium. Therefore, in order to provide additional micromechanical retention, we remove ready composed veneer from diestone model and carried to an air abrasion chamber to etch the internal surface of veneer.

The teeth were isolated with a rubber dam (Dental Dam, HYGIENIC, 6" X 6", 152X152 mm, Coltène/Whaledent Inc., 235 Ascot Parkway, Cuyahoga Falls, OH 44223 / USA) that is useful to keep the operative field dry; the rubber dam was fixed on each tooth with wires.

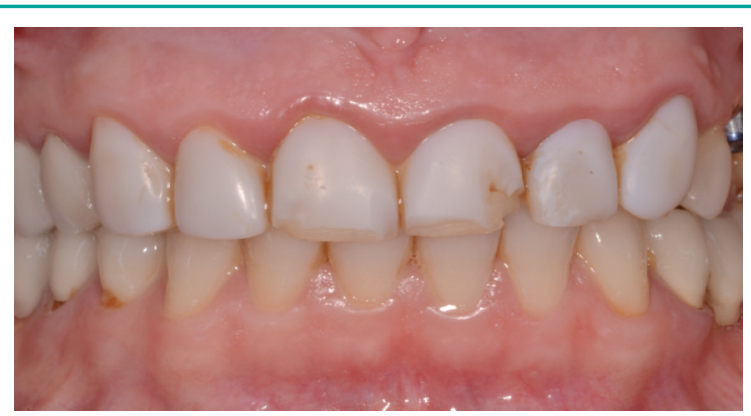

Figure 1

Pre-operative case.

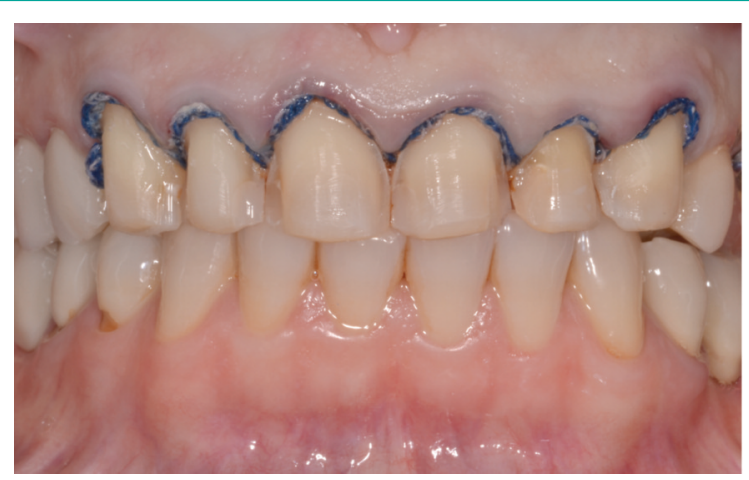

Figure 2

Tooth preparation before final impression. 
After etching using a $35 \%$ phosphoric acid gel with Benzalkonium Chloride for 20 seconds over the teeth surface, the teeth was then washed with an air water spray (Wet tecnique) and a thin layer of adhesive (Tokuyama Bond Force, Tokuyama Dental Corporation, 38-9, Taitou 1chome, Taitou-kuu, Tokyo, Japan) applied on the prepared teeth surface and light cured. Then we applied luting composite [Enacem UD4, MICERIUM S.p.A. Via Marconi 83 -16030Avegno (GE) Italy] to the internal surface of the veneer and the prepared tooth and removed the excess composite resin with a brush dipped in bonding agent before curing (Fig. 3). Other veneers were placed in same fashion. Static and dynamic occlusion was checked. The final polishing of restoration surface was carried out with silicon rubber. The case control after 1 year (Figs. 4, 5).

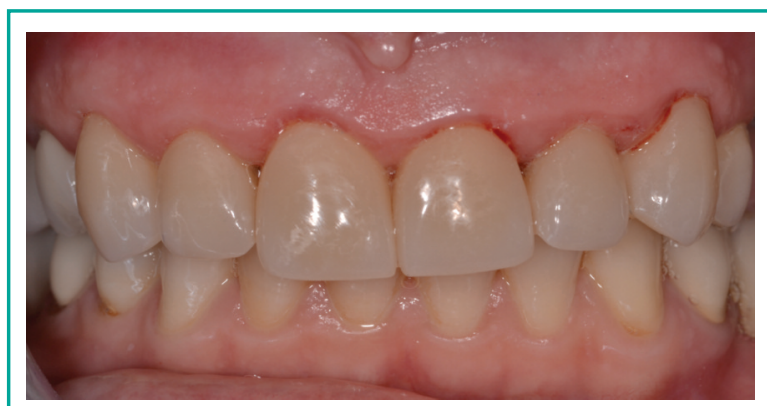

Figure 3

Veneers after cementation.

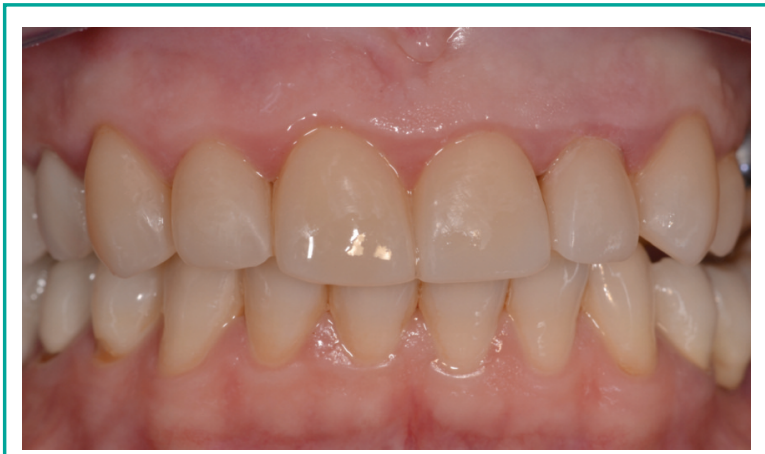

Figure 4

Case control after 1 year.

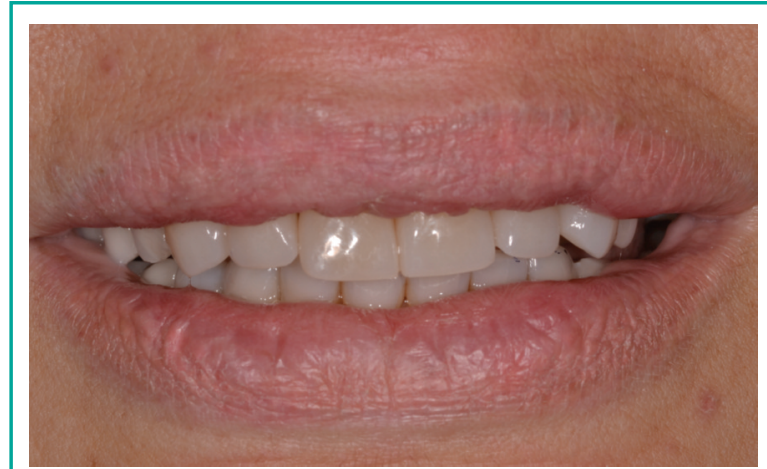

Figure 5

Case control after 1 year.

\section{Discussion and results}

While the invention of veneering anterior teeth by Dr. Pincus was presented in 1937, it became more popular in the mid-seventies, using three different approaches: direct bonding using resin composites, prefabricated composite veneers and indirect, custom-made veneers (4).

The advantages offered by indirect techniques compared to direct techniques are as follows:

- Superior aesthetic result

- Adequate abrasion resistance

- Biocompatibility with soft tissues

- Dimensional and chromatic stability over time

- Strong bond between the two adhesive interfaces (luting agent/etched enamel and luting agent/etched porcelain or postpolymerized resin composite).

Thanks to improved oral hygiene habits and the greater reliability of recent aesthetic materials, the indications for anterior indirect adhesive restorations have enlarged to cover most patients. Universally, the following clinical situations are considered indications for anterior indirect restorations:

- Enamel hypoplasia, waves, stains, grooves, etc.

- Enamel abrasions

- Congenital imperfect amelogenesis caused by hormones or tetracyclines

- Chromatic or distrophic alterations caused by fluorosis 
- Numerous aesthetically unsatisfactory superficial restorations

- Coronal fractures located primarily on the palatal side

- Volume anomalies (microdens)

- Diastemas

- Alignment defects (5).

The increased practice of veneering technique with indirect composites is due to improvement in the properties of composite materials in the last years. The mechanical and physical properties of veneering composite resins are based on their chemical composition: resin matrix, filler particle type, filler size, filler percentage, and filler-matrix bonding (silane coupling agent). Temperature, environmental conditions, and the light intensity of the polymerization unit are all important factors. In addition, longer light exposure and postcuring by heating have been found to improve the properties of prosthetic composite resin materials in laboratory studies. It has been shown that the failure probability of composite resin-veneered restorations was not significantly different from that of metal ceramic restorations (3).

The composition of these indirect veneering composites systems is similar to that of direct composites, differing by the method of additional polymerization. The veneering composite is layered in thin layers and each layer is then cured with special devices using light polymerization as well as heat curing, both in combinations with pressure/vacuum and/or nitrogen atmosphere (2). These restorations have proved durable and aesthetic, protect tooth structure and aesthetic and function is predictably re-established. The results were very favourable, and the patient was satisfied.

\section{Conclusions}

The demand for treatment of unesthetic teeth is steadily growing. Accordingly, several treatment options have been proposed to restore the esthetic appearance of teeth, like full-coverage crowns and bonding with composites. While full-coverage crowns is highly invasive and may have an adverse effect on the pulp or periodontal tissue, bonding with composites on the other hand, even though less invasive, continues to remain susceptible to discoloration, wear and marginal fractures. Laminate veneers were reported to provide a superior alternative to direct composite resin bonding for esthetic modification of teeth. These restorations offer a successful treatment that preserves tooth structure while providing excellent aesthetic results and patient acceptance $(5,6)$.

The indirect veneers have undergone considerable improvement and refinement over the past few decades, and have now matured into a predictable restorative concept in terms of longevity, periodontal response and patient satisfaction. These veneer restorations provide a valid conservative alternative to complete coverage as they avoid aggressive dental preparation; thus, maintaining tooth structure (5).

\section{References}

1. Korkut B, Yanıkoğlu F, Günday M. Direct composite laminate veneers: three case reports. JODDD. 2013; 7(2):105-111

2. Egli RE. Impact of in-vitro aging on mechanical and optical properties of veneering composites. 2010, University of Zurich, Faculty of Medicine. Postprint available at: http://www.zora.uzh.ch.

3. Stawarczyk B, Egli R, Malgorzata Roos, Mutlu Özcan, Hämmerle CHF. The impact of in vitro aging on the mechanical and optical properties of indirect veneering composite resins. The Journal of Prosthetic Dentistry. 2011;106(6):387-398.

4. Dietschi D, Devigus A. Prefabricated composite veneers: historical perspectives, indications and clinical application. Eur J Esthet Dent. 2011 Summer;6(2):178-87.

5. Shetty A, Kaiwar A, Shubhashini N, Ashwini P, Naveen DN, Adarsha MS, Shetty M, Meena N. Survival rates of porcelain laminate restoration based on different incisal preparation designs: An analysis. J Conserv Dent. 2011 Jan-Mar;14(1):10-15.

6. Asensio Acevedo R, Suarez-Feito JM, Suárez Tuero C, Jané L, Roig M. The use of indirect composite veneers to rehabilitate patients with dental erosion: a case report. Eur J Esthet Dent. 2013 Autumn;8(3):414-31.

Correspondence to:

Prof. Marco Gargari

E-mail:marco.gargari@gmail.com 